\title{
Circular Polarization Array Antenna with Triplate Stripline Elliptic Resonator Antennas
}

Yumi Takizawa

Institute of Statistical Mathematics

Research Organization of Information and Systems

Tokyo, Japan

takizawa@ism.ac.jp

Cahya Edi Santosa

Center for Environmental Remote Sensing

Chiba University, Chiba, Japan

maxedi77@gmail.com

\author{
Atsushi Fukasawa \\ Former Professor, Chiba University, and \\ Technical Adviser \\ Musasino Co. Ltd, Tokyo, Japan \\ fukasawafuji@yahoo.co.jp \\ Josaphat Tetuko Sri Sumantyo \\ Center for Environmental Remote Sensing \\ Chiba University, Chiba, Japan \\ jtetukoss@faculty.chiba-u.jp
}

\begin{abstract}
This paper presents a plane antenna and array for X-band circular polarization. The novel factors of this development are elliptic resonator antenna and Glass-Epoxy substrates. High efficiency in resonance and radiation of microwave energy is expected by elliptic resonators. Easy process in fabrication is expected by Glass-Epoxy substrate. Elliptic ratios of feed- and reactance-elements are chosen independently each other. The dielectric constant $\varepsilon_{r}$ and $\tan \delta$ are 4.6 and 0.010 respectively. Enough bandwidth of axial ratio of circular polarization and directive gain are confirmed to be $15 \%$ of central frequency and $10 \mathrm{~dB}$ respectively for antenna array.
\end{abstract}

Key-Words: - Circular polarization plane antenna, S-type routing wire, Reduction of horizontal radiation, Grounded square collar

Received: March 30, 2020. Revised: November 29, 2020. Accepted: December 8, 2020. Published: December 31, 2020.

\section{Introduction}

Stripline array antenna provides remote sensing systems with compact and inexpensive antennas. The authors are interested in three-layered substrates to compose wideband circular polarization plane antenna. Low loss stripline antennas have been designed using fluorine resin (Teflon) substrate conventionally. The permittivity (relative dielectric constant $\varepsilon r$ ) is small as 2.17 at $10 \mathrm{GHz} \mathrm{X}$-band, so the parameter values (practical dimensions) are not needed to be strict. Metallization and multilayered substrates of the Teflon require much processing cost and times.

In this development, two novel factors are considered. The first one is the structure of stripline resonators. Triplate stripline configuration is composed by feed-and reactance-elements on the ground plate. This concept was given by the structure of Yagi-Uda antenna[1]. This antenna is composed of three elements. Feed- and guide/radiation of microwave energy. Another element is assigned by reflector of microwave energy. Feed- and reactance-elements corresponds to feed- and guide/radiation elements. Another is assigned as the ground element.

For microwave circular polarization, degeneration of two resonant modes must be realized in a resonator.

Square and circular disc conductors with truncation are used degeneration in conventional antennas [2, 3].

In this paper, elliptic feed-and reactanceelements are proposed. Low and high frequency resonances are assigned to meet the required condition of degeneration.

The second factor of this development is dielectric material of Glass-Epoxy substrates. It is expected to provide easy process in fabrication. The relative dielectric constant $\varepsilon r$ is high 4.6, and $\tan \delta$ 0.01 . It is estimated that design rule is too strict compared to that of the antenna made of Teflon substrate. 


\section{Unit Antenna}

Figure 1 gives a triplate stripline resonator antenna composed of feed- and reactance-elements $a$ and $b$, and the ground plate $g$. The substrate $s$ under the ground plate provides feeding routing wire for the antenna. Feed element $a$ is fed with vertical probe through substrate $s$ under the ground plate. Collar $c$ shows a $\lambda g / 4$ line with short termination for suppression of horizontal radiation.

Figure 2 shows elliptic feed- and reactanceelements. They are defined by individual ellipses. 2ra1, $2 \mathrm{ra} 2$ are major and minor axes of ellipse of feed-element, which yields $x$ and $y$ axis-components for a rotating polarization vector.

\section{Four-Antenna Array}

\subsection{Orthogonal arrangement of four antennas}

An orthogonal arrangement of four-antenna array is given in Fig. 3. Each antenna is settled in the space of four quadrants in the coordinate $(X, Y)$.

The rotational directions of the four antennas are set in the rotational symmetry with 90 degree difference in each other.

Considering the clockwise circular polarization, each antenna in spaces advances 90 degree electrically.

\subsection{Compensation of the special and temporal phases}

Compensation of the special and temporal phases is needed to provide synchronous radiation among four antennas.

Considering the relations of the electrically advanced phase, these antennas should be fed with delay of 90 degree electrically to keep the condition of synchronous rotation field by four antennas based on.

\subsection{Parallel feeding}

Orthogonal arrangement of antenna array and parallel feeding have been designed by Y. Takizawa and A. Fukasawa.

One of the pair antenna is given by two antennas in the 1 st and the 4th quadrants. Another pair antennas is given by two antennas in the 2nd and the 3rd quadrants.

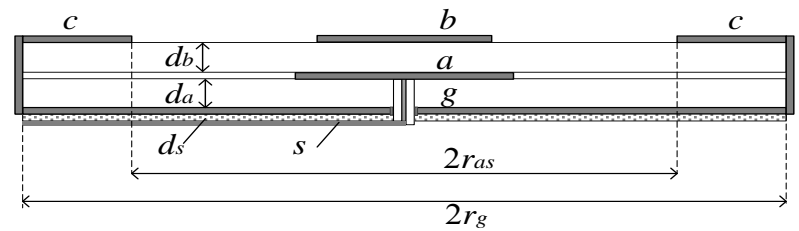

Fig. 1 Triplate stripline resonator antenna.

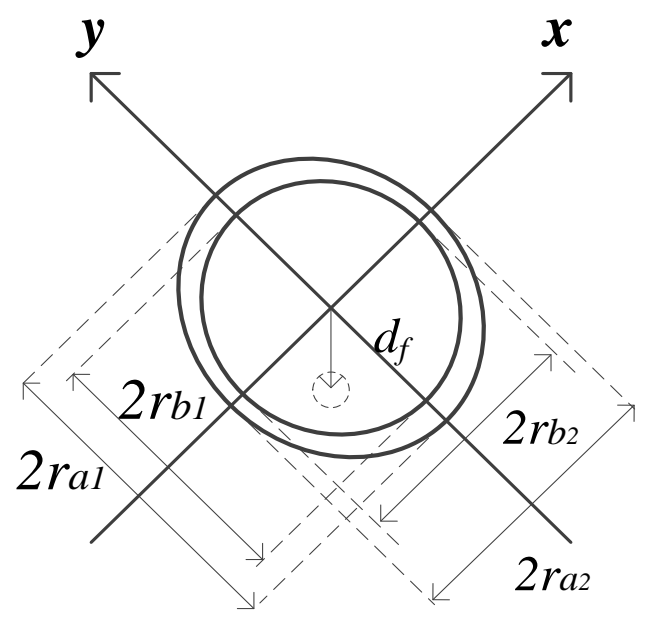

Fig. 2 Elliptic Feed- and reactance elements. Ground plate and grounded collar are abbreviated.

Parallel feeding is realized between the first and the second pair antennas. Right angle bending, Tbranches, and the other transmission lines with rapid bending cause so much reflections to deteriorate electrical characteristics of multiple antenna array.

A novel feeding wire given by Y. Takizawa and A. Fukasawa[6, 7] is shown in Fig. 3. Specific designed routing wires for parallel feeding circuit are composed of different S-type lines. In spite that lengths of $2 \mathrm{~S}$-curves are different, but the numbers of bending are set equal. This gives the minimum reflection caused by cancellation of the left and the right parts of array.

The dotted line in Fig. 3 shows a collar proposed by Y. Takizawa and A. Fukasawa[10]. It is composed of a quarter wavelength line with short termination at the peripheral of the array. It provides the function to suppress microwave radiation along horizontal directions. The suppressed microwave energy for horizontal direction ( $x-y$ plane) is added to radiation along vertical direction ( $z$ axis), and enhances directive gain of antenna array effectively.

The total numbers of antenna for an array is reduced almost half of the required number of design using conventional technologies. 


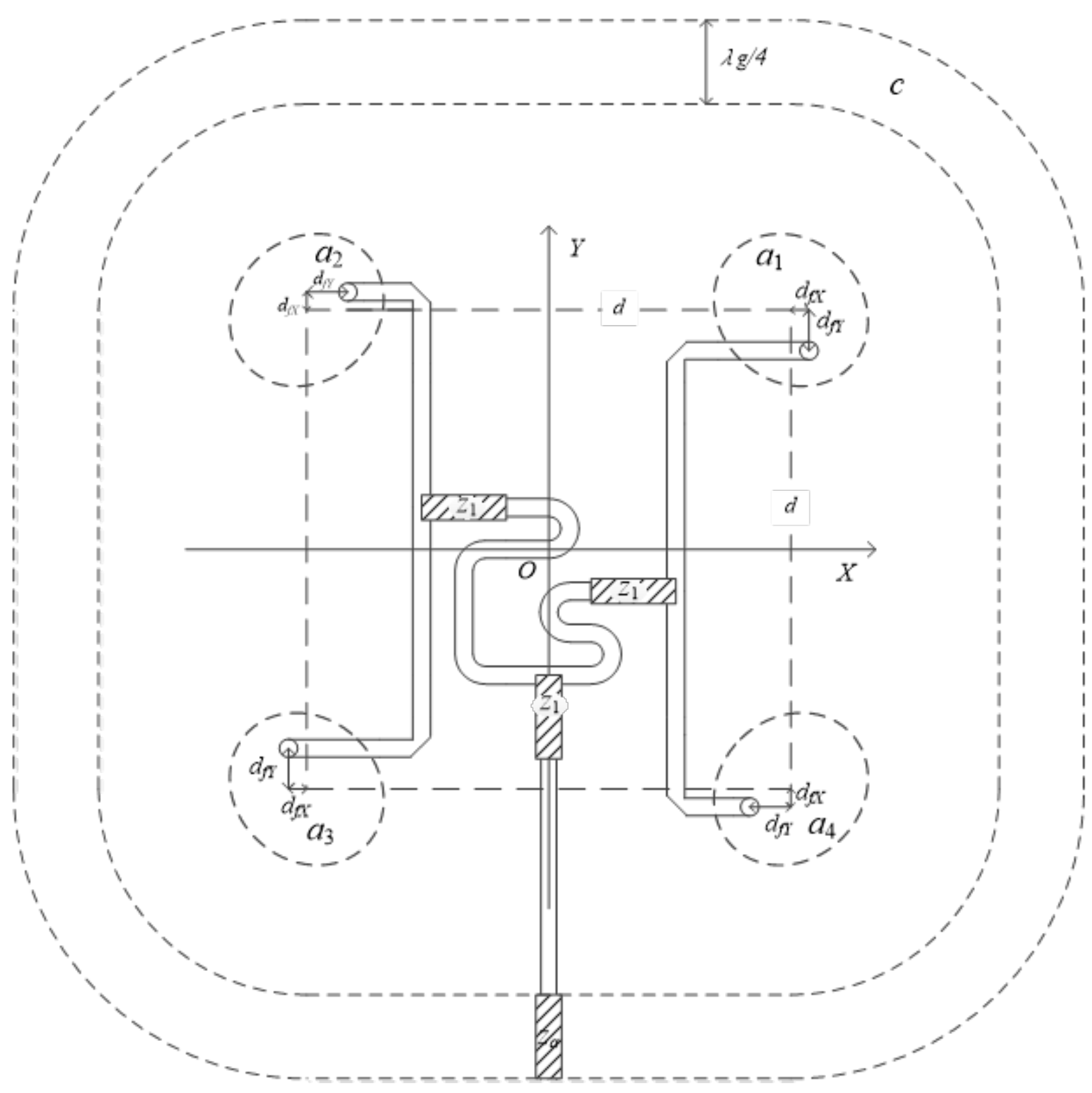

Fig. 3 Configuration of 4-antenna array and the feeding routing wires.

Dotted lines show a collar of short termination $\lambda \mathrm{g} / 4$ lines for suppression of horizontal radiation. $l x$ defines the position of the collar.

\subsection{Design of 4-antenna array}

(1) Design target

The central frequency and the bandwidth are given as follows;

Central frequency $\quad f 0=10 \mathrm{GHz}$

Bandwidth $\quad W=1 \mathrm{GHz}$
Glass-epoxy substrates are used, and the dimensions of the three-layer substrates are;

Dielectric substrate $\quad \varepsilon r=4.6, \tan \delta=0.010$

(2) Antenna Structure

Triplate stripline is composed as;

Thickness $(d a, d b, d s)=(1.0,1.0,0.4)$ 
(3) Array structure Antenna spacing $d=20(\mathrm{~mm})$

Array size including square collar $2 l g=42(\mathrm{~mm})$

(4) Antenna size and feed point

\begin{tabular}{|l|l|l|}
\hline parameter & dimension $(\mathrm{mm})$ & content \\
\hline $2 r a 1$ & 6.8 & feed element \\
\hline $2 r a 2$ & 5.9 & feed element \\
\hline $2 r b 1$ & 5.6 & reactance element \\
\hline $2 r b 2$ & 5.2 & reactance element \\
\hline$d f$ & 1.7 & feed point \\
\hline$r a 2 / r a 1$ & 0.87 & ellipticity $r a 2 / r a 1$ \\
\hline$r b 2 / r b 1$ & 0.93 & ellipticity $r b 2 / r b 1$ \\
\hline
\end{tabular}

Table 1 Parameter values antenna dimension.

\section{Characteristics of 4-Antenna Array}

The evaluation was given by 3D computer simulation CST in the environment constructed by C.E. Santosa, Chiba University. The characteristics of the array are evaluated by return loss, input impedance real and imaginary, axial ratio, and directive gain shown in Fig. 4, 5, 6, 7, and 8 . Wideband axial ratio and high directive gain were confirmed by this configuration and design.

Four different conditions in Fig. 4-7 correspond to different position of collars (quarter wavelength line termination at the peripheral of array) $l x=1.1 \sim$ $1.6(\mathrm{~mm})$. The best data was given by the blue lines $l x=1.1$ in Fig. 4-7, and red line in Fig. 8.

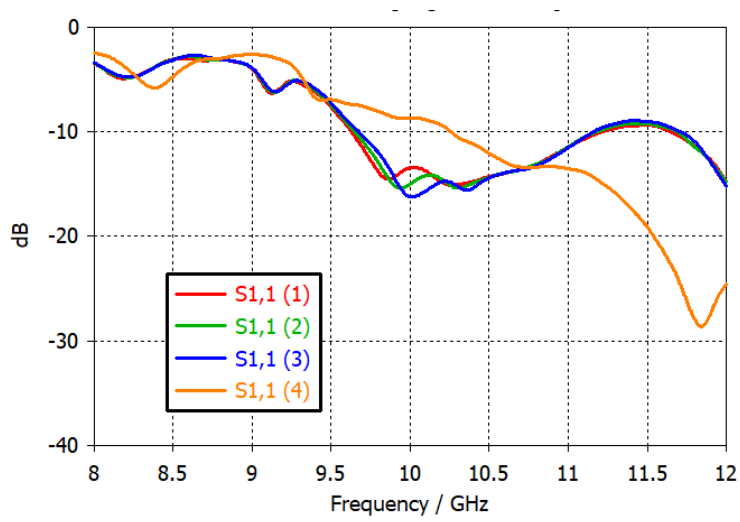

Fig. 4 Return loss

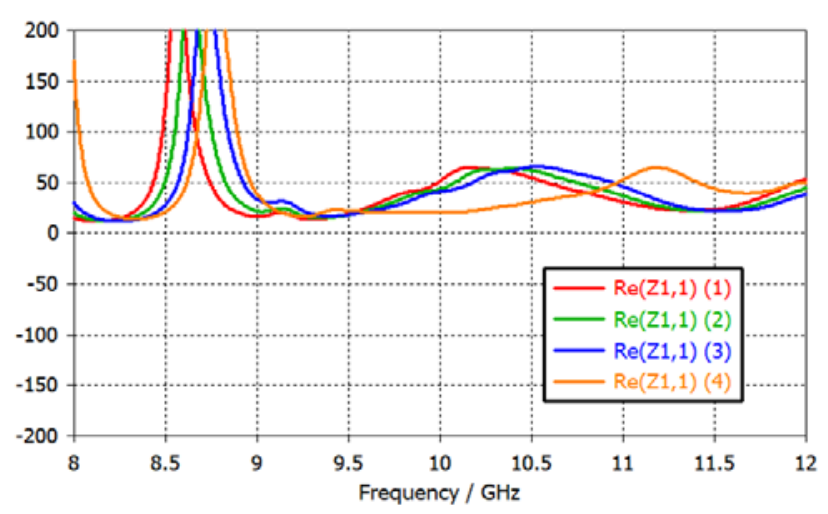

Fig. 5 Real part of Impedance.

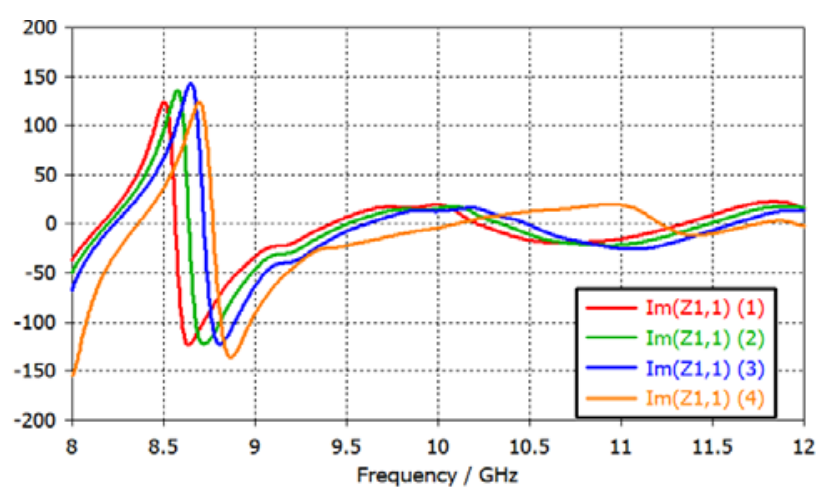

Fig. 6 Imaginary part of Impedance. 


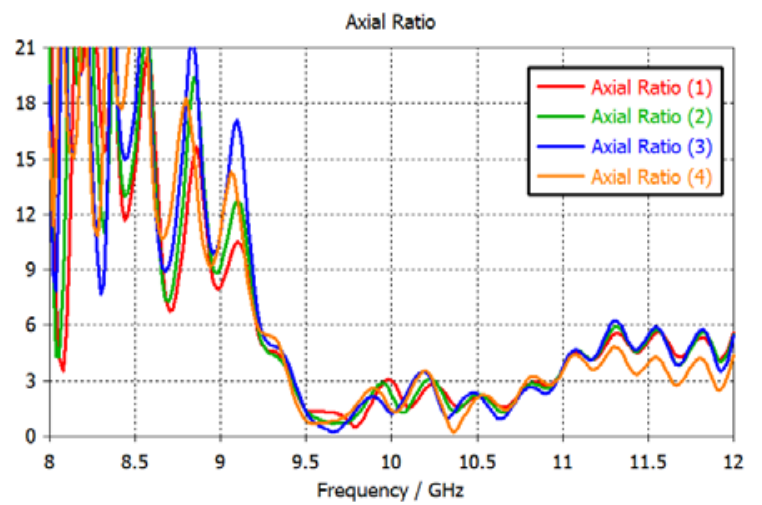

Fig. 7 Axial ratio of circular polarization.

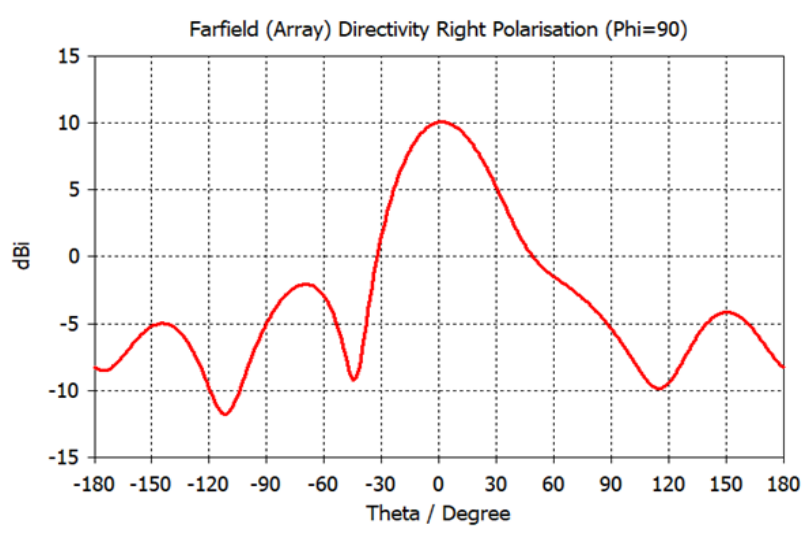

Fig. 8 Directive gain at $\varphi=90^{\circ}$.

\section{Conclusion}

The authors clarified the characteristics of an elliptic stripline resonator antenna and an array with four unit antennas. The unit antenna was composed of a triplate stripline resonator, whose feed- and reactance-elements were designed with independent ellipses.

The expected responses were confirmed with a four-antenna-array that the directive gain and the axial ratio were $10 \mathrm{~dB}$ and $15 \%$ of the center frequency. Relatively low directive gain was brought by the low tan $\delta$ of Glass-cloth substrates. Required precision and accuracy of design parameters to have sufficient performances are found almost equivalent between two different structures.

\section{Acknowledgment}

This work is supported by MEXT/JPS KAKENHI Grant Number 17K00067, and the scholarship donations given by Musasino Co. Ltd.

The authors express their sincere gratitude for effective supports and advices by the DirectorGeneral. Prof. H. Tsubaki, ISM and Prof. N. Kashiwagi, ISM.

And the authors express their sincere gratitude for kind supports by Mr. M. Abe, CEO, and Mr. M. Kise, General manager of R\&D, Musasino Co.Ltd.

\section{References:}

[1] Yagi S., Mushiake Y., Yagi-Uda Antenna, Sasaki Co., 1954.

[2] Haneishi M., et al,, Analysis, design, and measurement of small and low-profile antenna, Artech House (U.S.A), pp.1-270, 1991.

[3] Haneishi M., et al, Radiation properties of ringshaped microstrip antenna array, IEICE, Trans. on Communications, vol. E78-C, pp.995-1001, 1995.

[4] Takizawa Y., Fukasawa A., Microwave Patch Antenna with Circular Polarization for Environmental Measurement, Journal of Electromagnetics, vol. 2, pp.1-6, June 27, 2017.

[5] Takizawa Y., Fukasawa A., Novel Structure and the Characteristics of a $\mathrm{M}$ icrowave Circular Polarization Antenna, WSEAS Transaction on Communications, vol. 16, pp. 184-191, 2017.

[6] Takizawa Y., Fukasawa A., Circular Polarization Array Antenna with Orthogonal Arrangement and Parallel Feeding by Smoothed Routing Wires, Journal of Electromagnetics, Vol. 3, pp. 14-19, Apr. 11, 2018.

[7] Fukasawa A., Takizawa Y., Circular Polarization Array Antenna with Orthogonal Arrangement and Parallel Feeding by Simplified Routing Wires, Journal of Electromagnetics, Vol. 3, pp. 3 -8, Apr. 11, 2018.

[8] Takizawa Y., Fukasawa A., 16-Antenna Array for Circular Polarization with Wideband Axial Ratio and Enhanced Directivity, Journal of Electromagnetics, Vol. 3, pp. 20-26, Oct. 26, 2018.

[9] Takizawa Y., Fukasawa A., Circular Polarization Plane Antenna Array by AntiParallel Arrangement with Simplified Routing 
Wire, Journal of Electromagnetics, Vol. 3, pp. 27-32, Oct. 26, 2018.

[10] Takizawa Y., Fukasawa A.," 64-Antenna Array for Circular Polarization with Smoothed Routing Wires and Grounded Square Collar," Trans. on Communications, vol. 18, pp. 49-56, 2019.

[11] Takizawa Y., Fukasawa A., Santosa C.E., Sumantyo Josaphat T.S., Circular polarization antenna with elliptic feed- and reactanceelements using Glass Epoxy substrates, Journal of Electromagnetics, vol. 4, pp. 14-18, 2019.
Creative Commons Attribution License 4.0 (Attribution 4.0 International, CC BY 4.0)

This article is published under the terms of the Creative Commons Attribution License 4.0

https://creativecommons.org/licenses/by/4.0/deed.en_US 